Article

\title{
Fuzzy Logic-Based Model That Incorporates Personality Traits for Heterogeneous Pedestrians
}

\author{
Zhuxin Xue ${ }^{1,2}$, Qing Dong ${ }^{1}$, Xiangtao Fan ${ }^{1,3, *}$, Qingwen Jin ${ }^{1,2}$, Hongdeng Jian ${ }^{1}$ and Jian Liu ${ }^{1,3}$ \\ 1 Key Laboratory of Digital Earth Science, Institute of Remote Sensing and Digital Earth, Chinese Academy of \\ Sciences, Beijing 100094, China; xuezx@radi.ac.cn (Z.X.); dongqing@radi.ac.cn (Q.D.); jinqw@radi.ac.cn (Q.J.); \\ jianhd@radi.ac.cn (H.J.); liujian@radi.ac.cn (J.L.) \\ 2 University of Chinese Academy of Sciences, Beijing 100049, China \\ 3 Hainan Key Laboratory of Earth Observation, Sanya 572029, China \\ * Correspondence: fanxt@radi.ac.cn
}

Received: 15 September 2017; Accepted: 13 October 2017; Published: 20 October 2017

\begin{abstract}
Most models designed to simulate pedestrian dynamical behavior are based on the assumption that human decision-making can be described using precise values. This study proposes a new pedestrian model that incorporates fuzzy logic theory into a multi-agent system to address cognitive behavior that introduces uncertainty and imprecision during decision-making. We present a concept of decision preferences to represent the intrinsic control factors of decision-making. To realize the different decision preferences of heterogeneous pedestrians, the Five-Factor (OCEAN) personality model is introduced to model the psychological characteristics of individuals. Then, a fuzzy logic-based approach is adopted for mapping the relationships between the personality traits and the decision preferences. Finally, we have developed an application using our model to simulate pedestrian dynamical behavior in several normal or non-panic scenarios, including a single-exit room, a hallway with obstacles, and a narrowing passage. The effectiveness of the proposed model is validated with a user study. The results show that the proposed model can generate more reasonable and heterogeneous behavior in the simulation and indicate that individual personality has a noticeable effect on pedestrian dynamical behavior.
\end{abstract}

Keywords: pedestrian dynamical behavior; crowd simulation; fuzzy logic; personality trait; multi-agent

\section{Introduction}

Simulating crowd behavior as an interdisciplinary research field has attracted the keen interest of researchers and managers from various domains, including safety engineering, robotics, computer animation, and social psychology, and in recent years, it has been extensively studied and applied in these fields. For example, crowd simulation technology can be used to predict pedestrian flow [1-3] and recognize abnormal or normal behavior in safety engineering applications [4-6], and it has been implemented for autonomous navigation in robotics $[7,8]$ and enhancing the reality of computer animation [9-11]. The modelling of pedestrian dynamics is a common key issue among these applications.

Over the past three decades, various pedestrian models have been proposed to simulate realistic pedestrian dynamical behavior and understand the potential laws underlying complex crowd phenomena. In general, most of these models can be classified into two main types: macroscopic and microscopic. The former considers pedestrians as a fluid, and it usually uses Navier-Stokes or Boltzmann equations to represent the movement variations in density and speed [1,12]. The latter, which can analyse individual behavior and interactions among members of a crowd, describes a pedestrian as an object driven by rule [13] or force [2]. Compared with macroscopic models that focus on the trend of crowd movement, microscopic models can generate fine-grain simulation results to 
reflect individual issues and diversities. As a result, over the past few years, many researchers have paid more attention to microscopic modelling, such as the social force model [4], cellular automata model [3], and multi-agent model [14]. However, most microscopic models have been developed based on the assumption that human perception and decision-making in real time can be described using precise values, and they consider that the information obtained from environments can be accurately quantified in a pedestrian perception process and assume that the corresponding behavior can be predicted with certainty in the next decision process. In fact, this assumption does not conform with the nature of human behavior because imprecise concepts are attributes of human cognitive abilities. Therefore, a mathematic formulation with precise values cannot easily describe and predict realistic pedestrian dynamical behavior.

Inspired by soft computing theory, we attempt to incorporate a fuzzy logic system into a multi-agent simulation model to solve the above problem. The theory of fuzzy sets proposed by Zadeh [15] provides a useful modelling tool for many applications when ambiguity is present. For example, the traffic signal alternatives at a midblock crosswalk are controlled using fuzzy logic methods in [16,17], and a collision avoidance system for autonomous vehicles using a fuzzy steering controller is proposed in [8]. The perceptions and decisions of a pedestrian are usually represented by natural language, such as when the pedestrian attempts to pass through the exit at a fast speed rather than at $2 \mathrm{~m} / \mathrm{s}$. These processes can be formulated by a set of verbal variables and linguistic rules using a fuzzy logic system. Because of the advantages of accessible input perception-based information and easily steerable output, fuzzy logic-based methods have achieved great progress in the modelling of pedestrian dynamics. To the best of our knowledge, most studies using fuzzy logic have focused on the human ability to perceive their surrounding environments [18-24] and ignored decision-making processes that are vague and imprecise. Compared with previous works, we propose an approach to emulate pedestrian cognitive ability from a new perspective that uses the fuzzy logic system to model subjective decisions. Moreover, a concept of decision preferences is presented to represent the intrinsic control factors underlying decision-making.

Pedestrian dynamical behavior varies from one individual to another when they are confronted with similar situations in the real world. Thus, the modelling of heterogeneous behavior plays an important role in the simulation of a natural and realistic crowd. Among the many factors that promote variations in pedestrian behavior, such as physiological and psychological characteristics, personality has a significant impact on the subjective decision of the pedestrian. Personality is a pattern of behavioral, temperamental, emotional, and mental traits for an individual. People with various personalities have salient behavior characteristics. To generate the heterogeneity of the pedestrian cognitive ability, we introduce the well-known OCEAN (Openness, Conscientiousness, Extraversion, Agreeableness, and Neuroticism) personality model [25] to realize the different decision preferences of heterogeneous pedestrians in this paper.

The main contribution of this study is the proposal of a novel pedestrian model that incorporates fuzzy logic theory into a multi-agent system to address cognitive behavior that introduces uncertainty and imprecision in decision-making. For simulating the heterogeneous subjective decisions of pedestrians, the OCEAN personality model is introduced to generate the different decision preferences that represent the intrinsic control factors of decision-making. The experiments show that the application developed using our model can simulate more reasonable and heterogeneous pedestrian dynamical behavior in several normal or non-panic situations, including a single-exit room, a hallway with obstacles, and a narrowing passage. Furthermore, the results reveal the impact of personality traits on pedestrian dynamical behavior.

The remainder of this paper is organized as follows: Section 2 provides an overview of the related work. Section 3 describes the details of our model that incorporates fuzzy logic into a multi-agent system and uses personality traits for heterogeneous pedestrians. Section 4 presents the simulations and validation of the proposed model. Finally, Section 5 concludes the paper with a summary and outlook. 


\section{Related Work}

Over the years, various microscopic models have been presented to simulate pedestrian dynamical behavior under abnormal and normal situations. These models are usually classified into three types: cellular automata, social force, and multi-agent. The cellular automata model $[3,26,27]$ describes pedestrian flow through a discrete arrangement of space into grids of equal cells that have two states: occupied or unoccupied. According to a set of simple transition rules, the state of cells can be updated to indicate the movement of pedestrians at each time step. However, the pedestrians in this model move in discrete time and space; therefore, the simulation result is highly dependent on the discretization level of time and space, and it is difficult to ignore pedestrian body size and step time to accurately simulate pedestrian dynamical behaviors. The social force model $[2,4]$ inspired by Newton's second law describes the pedestrians' behavior with a mathematical equation of the interaction forces that consist of socio-psychological and physical forces. This model can easily reproduce certain self-organization phenomena in a real evacuation, such as arching, clogging, and the "faster-is-slower effect". However, the computational complexity of the model rapidly increases with the crowd number, and heterogeneous pedestrian behavior is difficult to replicate. The multi-agent model $[6,14,28]$ proposes a computational methodology in which all individuals are modelled as autonomous agents that are capable of interacting with each other. Agents are autonomous software entities with the perception and social ability to perform goal-directed knowledge processing. The key advantage of a multi-agent system is that it can model the dynamics of real-life complex systems. Therefore, these systems are particularly suitable for simulating the cognitive process and behavior of pedestrians. Based on the above discussion, we focus on the multi-agent model for pedestrian modelling.

To improve the believability of crowd simulations, simulating the uncertainty and imprecision of human behavior is an important aspect in many scenarios, such as pedestrian steering and emergency evacuation. A fuzzy logic approach has certain advantages over other approaches, such as its ability to use perceptual information and human experience and knowledge, and to emulate human thought processes. Therefore, this approach represents a natural and suitable tool for modelling pedestrian dynamic behavior. Nasir et al. [18] introduced a fuzzy logic framework to predict the impact of perceived attractive and repulsive stimuli, within the pedestrian's field of view, on movement direction during normal situations. Zhu et al. [19] integrated fuzzy logic with the social force model and reproduced the dynamical features of pedestrian evacuation. Li et al. [20] presented a fuzzy logic-based approach for crowd simulation that extracts fuzzy rules from the realistic videos that can be considered a parameterized behavior model. Dell'Orco et al. [21] proposed a microscopic model of crowd evacuation defined on a continuous space and used a fuzzy logic technique to reproduce human reasoning. Nasir et al. [22] proposed a genetic fuzzy system to model and simulate a pedestrian's steering behavior in a built environment. Fu et al. [23] proposed a fuzzy theory-based behavioral model to investigate evacuation dynamics in a cellular space. Zhou et al. [24] proposed a fuzzy logic approach for simulating pedestrian dynamical behavior, and it integrates the intermediate results of local obstacle-avoiding behavior, regional path-searching behavior and global goal-seek behavior with mutable weighting factors. Although the above studies have achieved positive results for pedestrian dynamics using a fuzzy logic-based approach, the human ability to perceive external information is considered fuzzy, and the subjective differences among crowds is ignored because all individuals are treated as homogeneous. Therefore, the differences in decision-making for heterogeneous individuals are difficult to simulate in these studies.

In order to simulate the different decision-making of heterogeneous pedestrians, researchers have also proposed some approaches using fuzzy logic theory. Akasaka et al. [29] presented a pedestrian navigation system using fuzzy measures and integrals for selecting a route based on users' own preference for routes. Teknomo et al. [30] integrated multi-states of pedestrian situation and pedestrian group behavior into the multi-agent pedestrian simulation through the concept of fuzzy inter-personal spacing. These studies directly simulated pedestrian navigation under some certain conditions from the 
perspective of subjective selection, but neglected the intrinsic factors influencing pedestrian behavior. In this paper, we attempt to model individual intrinsic characteristics for realizing the different decision preferences of heterogeneous pedestrians. The behavior of a pedestrian can be mainly influenced by physiological and psychological characteristics. For the aspect of physiology, Zheng et al. [9] chose four basic physiological characteristics - gender, age, health, and body shape-to generate heterogeneous crowd behavior. In terms of psychological characteristics, Durupinar et al. [10] integrated the OCEAN personality model into HiDAC (High-Density Autonomous) [31] to simulate crowd behavior by mapping between personality traits and observed behavior types. Guy et al. [11] presented an approach to simulating a heterogeneous crowd using the PEN (Psychoticism, Extraversion, and Neuroticism) personality model based on the RVO (Reciprocal Velocity Obstacle) library [7]. These studies showed that the modelling of pedestrian characteristics plays an important role in simulating realistic and heterogeneous pedestrian dynamical behavior. However, they are based on the assumption that human decision-making can be described using precise values. To solve the problem of uncertainty and imprecision during decision-making, we adopt a fuzzy logic-based approach to map the relationships between the personality traits and the decision preferences.

In this paper, we propose a new pedestrian model that incorporates fuzzy logic theory into a multi-agent system. Our model can emulate pedestrian cognitive ability that introduces uncertainty and imprecision during decision-making. Inspired by previous works, we choose the OCEAN personality model to simulate the individual characteristics for generating the different decision preferences of heterogeneous pedestrians. To describe the fuzzy attributes, a fuzzy inference system is adopted for mapping the relationships between the personality traits and the decision preferences.

\section{Model Description}

In this section, we first introduce an overview of our proposed model for simulating microscopic pedestrian behavior based on multi-agent systems. Next, we describe the well-known Five-Factor personality model, which is also known as the OCEAN model and used in this study to drive the heterogeneous behavior of individuals within a crowd. Then, we present a concept of decision preferences and define the meanings of these parameters. Finally, a fuzzy logic-based approach is presented to represent the relationship between the personality traits and the decision preferences of agents for addressing uncertainty and imprecision during decision-making.

\subsection{Overview of the Proposed Model}

Human behavior is a complex phenomenon that is difficult to capture via computers performing mathematical equations. In addition, crowd behavior is not a simple collection of individual behaviors in the crowd but also includes the interactions between people. To generate heterogeneous crowd behavior and understand the complicated motion features of pedestrians, we adopt a framework based on a multi-agent system to model and simulate pedestrian movement. A multi-agent system is an extension of agent technology where a group of loosely connected autonomous agents act in an environment to achieve a common goal [32]. In this framework, each human individual is modelled as an autonomous agent who interacts with a virtual environment and other agents. We believe that such a framework is particularly suitable for simulating individual cognitive processes and behavior in pedestrian dynamics.

In our proposed simulation framework, the virtual environment mainly includes agents, obstacles, and open spaces. The agent consists of three basic components: perception module, decision-making module, and action module.

- Perception module: This module of an autonomous agent is included to perceive the surrounding information from the virtual environment, and it has an important role as the information portal of the agent that interacts with the external environment. The perceived information of an agent mainly includes (1) the location of other agents and obstacles; (2) the distance from itself to other 
agents and obstacles; (3) the speed of other agents; and (4) the range of obstacles. Because of the limited range of the visual field, each agent has a limited sensing capability.

- Decision-making module: This module is designed to represent the cognitive and reasoning processes associated with the movement of a pedestrian. The perceived information is used as input in this module for an agent making decisions on steering behavior. The publicly available RVO2 library is used to implement the reasoning process, and the decision preferences are defined using the following five parameters: (1) NeighborDist; (2) MaxNeighbors; (3) TimeHorizon; (4) Radius; and (5) PrefVelocity. A detailed description of these parameters is presented in Section 3.3. The multi-agent system consists of heterogeneous agents, or agents with different decision-making capabilities.

- Action module: In this module, the actions of an agent include the agent's ability to walk, run, turn, and stop, which are the basic locomotion capacities of a pedestrian in a real-life environment. In this article, we use two main variables - the speed and direction of movement- to express the basic actions in the steering activity. Actions driven by decision-making can vary considerably among agents presented with the same perceived information.

To simulate the uncertainty and imprecision of pedestrian dynamical behavior, we incorporate a fuzzy logic system into a multi-agent simulation framework to model the decision-making process of an agent. Compared with previous works, we focus on the different decision-making abilities of heterogeneous agents in this paper. For the sake of simplicity, we use the decision preferences as fuzzy variables to describe qualitative cognitive behaviors in pedestrian decision-making. According to the fuzzy relationship between personality traits and human behavior [33], we introduce the OCEAN personality model to represent heterogeneous pedestrians with various characteristics, such as openness, conscientiousness, extraversion, agreeableness, and neuroticism. In addition, the mapping between personality factors and decision preferences is determined by a fuzzy inference system.

The overall framework of our proposed model is shown in Figure 1. In the following sections, we describe the personality model, decision parameters, and a fuzzy logic-based approach in more detail.

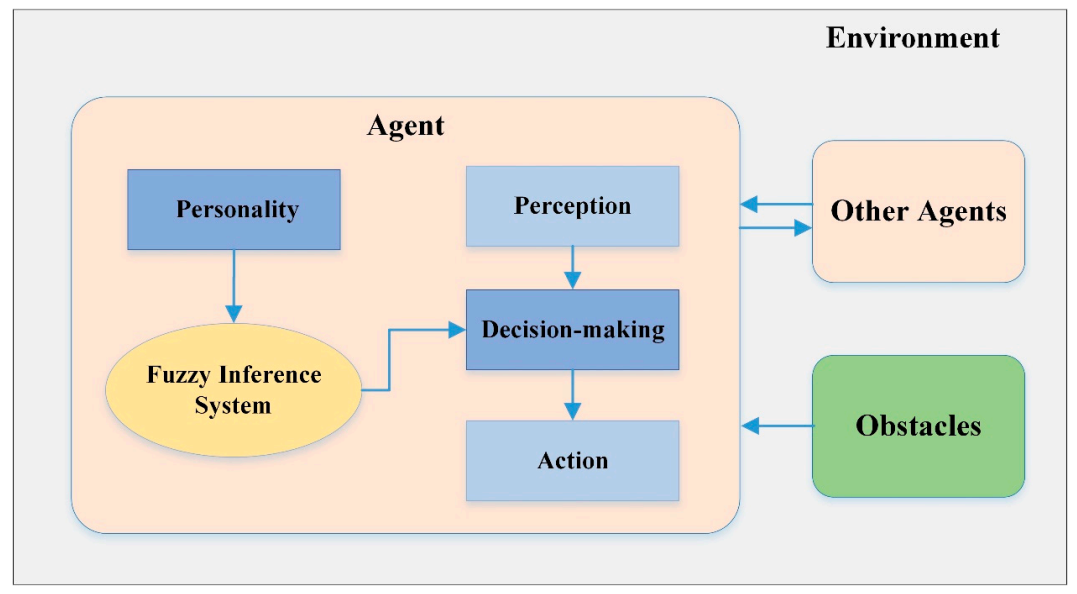

Figure 1. Framework of the proposed model based on a multi-agent system.

\subsection{Modelling of Personality}

Research in social science and human psychology defines personality as representing the pattern of a person's thoughts, feelings, and behaviors, which distinguish one person from another and persist over time and situations [34]. To exhibit a natural and realistic simulation effect, personalities should be considered an important aspect of modelling heterogeneous pedestrian behavior because pedestrians with different personalities may react differently to the same situation. In addition, understanding 
how varying personality factors affect the walking behaviors of pedestrians is important for many applications.

Many mature models have been proposed by psychologists to describe the spectrum of personalities, such as Eysenck's three-factor model [35] and the Five-Factor model [25]. In this paper, we choose the famous Five-Factor personality model, which is also known as the OCEAN model, to characterize the personality traits of autonomous agents. This model categorizes personality into five orthogonal factors based on a factor analysis of user studies in which participants use common language adjectives to describe the behavior of people. The five factors have been defined as Openness, Conscientiousness, Extraversion, Agreeableness, and Neuroticism [36]. These factors are explained as follows:

- Openness reflects the degree of curiosity and creativity and preferences for novelty and variety.

- Conscientiousness describes the level of organization and care exhibited in collective activities.

- Extraversion is related to the degree of energy, sociability, and outgoingness.

- Agreeableness is a tendency to exhibit compassion and cooperation rather than suspicion and antagonism towards others.

- Neuroticism is the tendency to experience unpleasant emotions easily, such as anger, anxiety, depression, or vulnerability, and is the opposite of emotional stability.

To satisfy the goal of generating realistic and heterogeneous crowd behavior, we must model all agents with either salient characteristics or non-characteristics. Each factor of the OCEAN model is bipolar and consists of several traits [37]. We divide each factor into three types, namely, negative, neutral, and positive types, and describe them via descriptive adjectives. The details of the personality factors in our study are given in Table 1 , where,$-=$, and + mean negative, neutral and positive.

Table 1. Personality factors-Hierarchical types-Descriptions of characteristic.

\begin{tabular}{ccc}
\hline Factor & Type & Characteristic \\
\hline \multirow{3}{*}{ Openness } & - & cautious, narrow, conservative \\
& $=$ & occasional curiosity, moderate creativity \\
& + & curious, inventive, explorer \\
\hline \multirow{3}{*}{ Conscientiousness } & - & careless, rude, changeable \\
& $=$ & spontaneous, reasonable order \\
& + & persistent, organized, dependable \\
\hline Extraversion & - & shy, withdrawn, introvert \\
& $=$ & neutral, self-conscious \\
Agreeableness & + & social, energetic, outgoing \\
\hline Neuroticism & - & competitive, negative, harsh \\
& $=$ & somewhat gentle, moderately tolerant \\
& + & cooperative, compassionate, friendly \\
\hline & $=$ & calm, secure, confident \\
& + & occasional anxiety, basically stable \\
& & sensitive, fearful, nervous \\
\hline
\end{tabular}

This model describes the personality characteristics using a set of five factors rather than separate factors. Therefore, we use a five-dimension vector to model an agent's personality $P$ :

$$
\begin{gathered}
P=(V(O), V(C), V(E), V(A), V(N)) \\
V(i) \in[-100,100], i \in\{O, C, E, A, N\}
\end{gathered}
$$

where $O, C, E, A$, and $N$ represent Openness, Conscientiousness, Extroversion, Agreeableness, and Neuroticism, respectively. The default personality of agents is configured with $P=(0,0,0,0,0)$. 


\subsection{Decision Preferences}

Our main goal is to generate realistic and natural pedestrian dynamic behavior by simulating human cognitive ability with uncertainty and imprecision in movement, and the critical step is determining the method of describing the subjective decision-making of pedestrians. We consider that the parameters representing the influence factors of the decision must satisfy the following requirements:

- The content of these parameters can be associated with the perceptual information obtained from the environment;

- The personality of an individual has a significant impact on the range of these parameters;

- These parameters can directly reflect the differences in decision-making between pedestrians.

To meet the above requirements, we present the concept of decision preferences to represent the intrinsic control factors of decision-making. In this paper, we choose five parameters-NeighborDist, MaxNeighbors, TimeHorizon, Radius, and PrefVelocity - as the decision preferences based on the RVO2 library. The RVO2 library provides an easy-to-use implementation of the optimal reciprocal collision avoidance (ORCA) formulation [38] for multi-agent simulations. Recently, many multi-agent-based approaches have used these or similar parameters to calculate the locomotion of agents in pedestrian simulation scenarios. These parameters are described below.

- NeighborDist is the maximal distance of other agents that the agent considers during path-planning. Here, we use this parameter to represent the spatial scope that the agent must consider when making decisions.

- MaxNeighbors is the maximum number of neighbours affecting the steering behavior of an agent. These neighbours are selected by the rule of nearest in spatial distance. The mutual interactions between agents are an important aspect of the simulation in this study. We use MaxNeighbors to describe whether the movement of an agent is susceptible to other agents.

- TimeHorizon is the minimal amount of time for which the simulated agent velocities are safe with respect to other agents and obstacles. This parameter is used to describe the planning horizon in the path-planning process. Larger TimeHorizon values correspond to greater foresight of the agent.

- Radius is the personal space maintained by the agent to avoid collisions in motion. Agents attempt to preserve this space when other agents are around. In normal circumstances, the agent also tends to wait for available personal space before moving.

- PrefVelocity is the preferred velocity of the agent that is used if no other agents or obstacles are present. We use this parameter to express the expectation of the pedestrian to achieve the goal. Different individuals may have different velocities when faced with the same situation.

Guy et al. [11] studied the mapping from simulation parameters to the perceived behavior of agents, and they obtained a range of effective parameter values via a data analysis. In this paper, we use their results as empirical data to define the range of the decision preferences. The range and default values of these parameters are shown in Table 2.

Table 2. Range of the decision preferences and their default values.

\begin{tabular}{ccccc}
\hline \multirow{2}{*}{ Parameters } & \multicolumn{3}{c}{ Value } & \multirow{2}{*}{ Unit } \\
\cline { 2 - 4 } & Min & Max & Default & \\
\hline NeighborDist & 3 & 30 & 15 & $\mathrm{~m}$ \\
MaxNeighbors & 1 & 100 & 10 & $(\mathrm{n} / \mathrm{a})$ \\
TimeHorizon & 1 & 30 & 10 & $\mathrm{~s}$ \\
Radius & 0.3 & 2.0 & 1.0 & $\mathrm{~m}$ \\
PrefVelocity & 1.2 & 2.2 & 1.45 & $\mathrm{~m} / \mathrm{s}$ \\
\hline
\end{tabular}




\subsection{Fuzzy Inference System}

In most decision-making processes, the capacity for addressing uncertainty and imprecision is a key issue and influences the quality of the decisions. The decision preferences reflect the cognitive ability of an individual with subjective differences. Precisely determining the values of these parameters is difficult because imprecise concepts are attributes of human cognitive abilities. For example, pedestrians are frequently described as attempting to move at a 'fast' speed rather than at ' $2 \mathrm{~m} / \mathrm{s}$ '. In addition, the OCEAN personality model introduced in Section 3.2 is defined based on linguistic variables. Compared with the traditional methods $[10,11]$, the mapping relationships with uncertainty and imprecision between the personality traits and the decision preferences can be easily determined.

In this paper, we incorporate a fuzzy logic system into the multi-agent simulation framework to handle the imprecise and uncertain issues of cognitive abilities on pedestrian dynamics. The fuzzy inference process includes four parts as follows:

- Under fuzzification, a crisp value of input variables can be transformed into a fuzzy value with membership degree by their membership functions;

- A rule base is the collection of the domain expert knowledge, and it is usually expressed as a set of 'IF-THEN' rules that are used to capture the relationship between inputs and outputs;

- During fuzzy inference, various fuzzy logic operations are used, and all fuzzy rules are triggered and combined to acquire a fuzzy consequence for each output variable;

- Under defuzzification, each fuzzy consequence must be transformed into a crisp value before the results can be used in simulation.

The structure of the proposed fuzzy logic system is shown in Figure 2, and more details are presented in the following section.

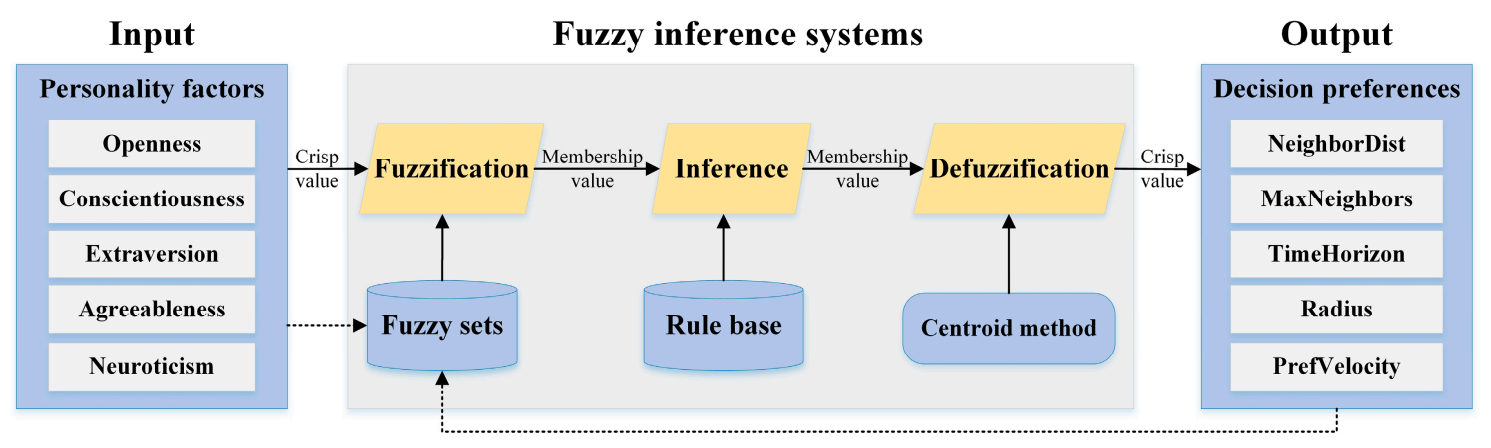

Figure 2. Structure of the proposed fuzzy logic system.

\subsubsection{Fuzzy Membership Functions}

In a fuzzy logic system, fuzzy sets and their membership functions should be defined for each input and output. Let $U$ represent the universe of discourse, with elements of $U$ denoted by $x$. A set $A$ is a fuzzy subset of $U$. The degree to which an element $x$ belongs to set $A$, which is a real number between 0 and 1 , is called the membership value $A(x)$ in the fuzzy set $A$. The meaning of a fuzzy set $A$ is characterized by a membership function $\mu_{A}$ that maps elements of a universe of discourse $U$ to their corresponding membership values $A(x)$ :

$$
A(x)=\mu_{A}(x) \in[0,1], x \in U .
$$

The fuzzy membership function $\mu$ can be represented by a variety of shapes, such as triangles and trapezoids, depending on how the expert relates different domain values to belief values.

In our proposed fuzzy logic-based approach, we consider each of the personality factors and decision preferences as linguistic variables. The inputs are the OCEAN personality factors Openness, 
Conscientiousness, Extraversion, Agreeableness, and Neuroticism. The outputs are the decision preferences NeighborDist, MaxNeighbors, TimeHorizon, Radius, and PrefVelocity. According to the description of the personality factors in Section 3.2 and the decision preferences in Section 3.3, we determine fuzzy sets of inputs and outputs as shown in Table 3, and their corresponding fuzzy membership functions are shown in Figure 3.

Table 3. Fuzzy attributes of inputs and outputs.

\begin{tabular}{|c|c|c|c|c|}
\hline & Name & Universe of Discourse & Fuzzy Sets & Membership Function \\
\hline \multirow[t]{3}{*}{ Inputs } & Each factor of OCEAN & {$[-100,100]$} & Negative & Trapezoidal \\
\hline & & & Neutral & Trapezoidal \\
\hline & & & Positive & Trapezoidal \\
\hline \multirow[t]{15}{*}{ Outputs } & NeighborDist & {$[3,30]$} & Near & Trapezoidal \\
\hline & & & Moderate & Trapezoidal \\
\hline & & & Far & Trapezoidal \\
\hline & MaxNeighbors & {$[1,100]$} & Small & Trapezoidal \\
\hline & & & Medium & Trapezoidal \\
\hline & & & Large & Trapezoidal \\
\hline & TimeHorizon & {$[1,30]$} & Short & Trapezoidal \\
\hline & & & Moderate & Triangular \\
\hline & & & Long & Trapezoidal \\
\hline & Radius & {$[0.3,2.0]$} & Small & Trapezoidal \\
\hline & & & Medium & Triangular \\
\hline & & & Large & Trapezoidal \\
\hline & PrefVelocity & {$[1.2,2.2]$} & Slow & Triangular \\
\hline & & & Moderate & Triangular \\
\hline & & & Fast & Trapezoidal \\
\hline
\end{tabular}

a

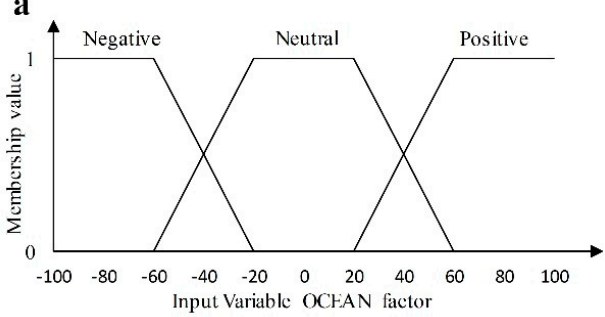

c

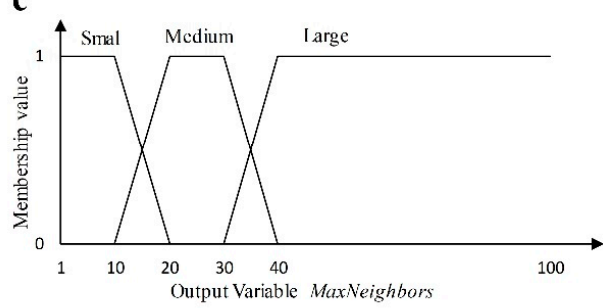

$\mathbf{e}$

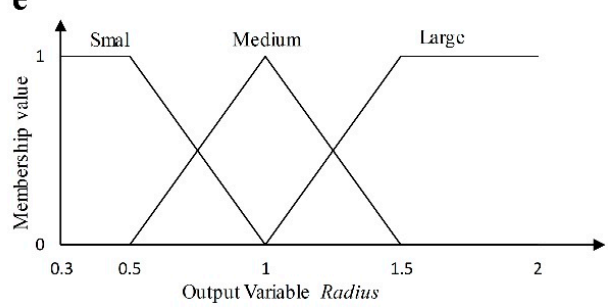

b

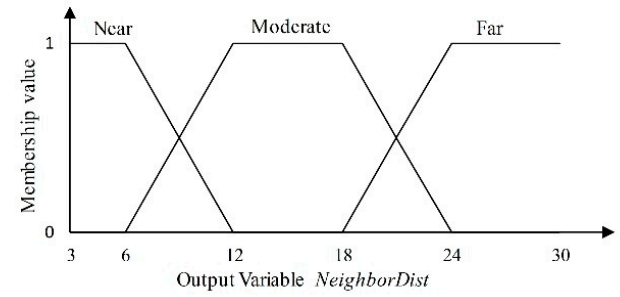

d

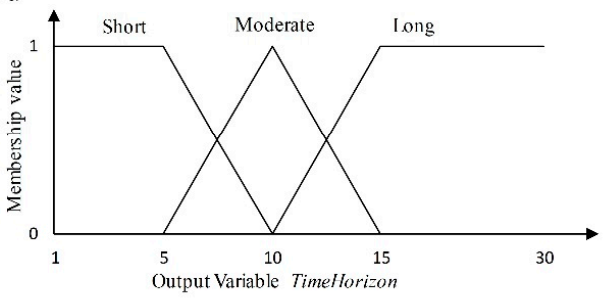

f

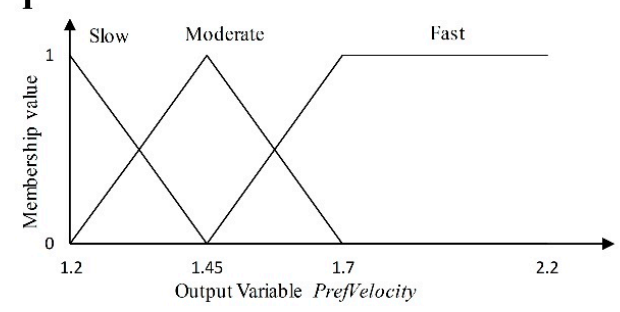

Figure 3. Membership function definition for the input and output variables: (a) each factor of the OCEAN personality model; (b) NeighborDist; (c) MaxNeighbors; (d) TimeHorizon; (e) Radius; (f) PrefVelocity. 


\subsubsection{Fuzzy Rules}

As the most widely applied reasoning pattern, a set of 'IF-THEN' rules are used by the fuzzy rule-based method to obtain the relationship between inputs and outputs. The antecedent of a fuzzy rule is a logical combination of fuzzy propositions, which is usually in the form of ' $x$ is $A^{\prime}$. The consequence of a fuzzy rule is calculated by the degree to which the antecedent is satisfied. In general, fuzzy rules are formulated by the domain expert based on empirical knowledge, and they can be gradually improved with further use. An example of the 'IF-THEN' fuzzy rule is as follows:

IF (Extraversion is Positive) AND (Agreeableness is Positive) THEN Radius is small.

The accuracy of a fuzzy inference system is affected by the number of linguistic fuzzy sets that cover the universe of discourse. However, the size of fuzzy rules grows exponentially with the number of input fuzzy sets of the antecedent. To obtain a trade-off between the computational complexity and accuracy, we select two personality factors as the major impact factors for each decision parameter. In this study, we construct a rule base containing fuzzy rules on the relationship between the OCEAN personality factors and the decision preferences based on previous studies $[10,11]$. The details are summarized as follows:

- NeighborDist is related to Openness and Conscientiousness. Pedestrians with positive Openness are more curious about their surroundings; therefore, they explore a larger scope in the path-planning process. Pedestrians who have a positive Conscientiousness trait notice individuals in the distance because they are predictable and self-disciplined.

- MaxNeighbors is related to Openness and Neuroticism. Pedestrians with positive Openness are likely to observe the behavior of more people around them. An important facet of the Neuroticism factor is sensitivity, with sensitive individuals more easily affected by others.

- TimeHorizon is related to Conscientiousness and Agreeableness. To maintain the orderly pedestrian flow, individuals with positive Conscientiousness tend to be prepared for upcoming events in advance. The Agreeableness factor describes the cooperative tendency of people. Pedestrians with a stronger cooperative tendency will respond earlier to avoid a collision during path-planning.

- Radius is related to Extraversion and Agreeableness. Individuals with positive Extraversion are outgoing and sociable and maintain a small territory in which they feel comfortable. Friendly individuals who have a positive Agreeableness trait usually do not react harshly when others are too close.

- PrefVelocity is related to Extraversion and Neuroticism. An individual's energy level is the key factor that determines their preferred velocity. In generally, extroverts tend to be more energetic and thus have a fast PrefVelocity, whereas introverts are more lethargic and present opposite characteristics to that of extroverts. Pedestrians with positive Neuroticism are prone to be anxious and tense when congestion occurs; therefore, they try to pass at a fast speed.

Because the antecedent has two input variables and each personality factor has three fuzzy sets, a total of 45 (or $5 \times 3^{2}$ ) 'IF-THEN' rules are established to deduce the decision preferences of pedestrian dynamical behavior. In Table 4, we report several sample fuzzy rules. 
Table 4. 'IF-THEN' fuzzy rules for the decision preferences.

\begin{tabular}{|c|c|c|}
\hline \multirow{2}{*}{ Rule Number } & \multicolumn{2}{|c|}{ IF-THEN Statements } \\
\hline & Antecedent & Consequence \\
\hline $\mathrm{R}_{1}$ & IF ( $O$ is Negative) AND ( $C$ is Negative) & THEN NeighborDist is Near \\
\hline $\mathrm{R}_{2}$ & IF ( $O$ is Negative) AND ( $C$ is Neutral) & THEN NeighborDist is Near \\
\hline $\mathrm{R}_{3}$ & IF ( $O$ is Negative) AND ( $C$ is Positive) & THEN NeighborDist is Moderate \\
\hline $\mathrm{R}_{4}$ & IF ( $O$ is Neutral) AND ( $C$ is Negative) & THEN NeighborDist is Moderate \\
\hline $\mathrm{R}_{5}$ & IF ( $O$ is Neutral) AND ( $C$ is Neutral) & THEN NeighborDist is Moderate \\
\hline $\mathrm{R}_{6}$ & IF ( $O$ is Neutral) AND ( $C$ is Positive) & THEN NeighborDist is Far \\
\hline $\mathrm{R}_{7}$ & IF ( $O$ is Positive) AND ( $C$ is Negative) & THEN NeighborDist is Moderate \\
\hline $\mathrm{R}_{8}$ & IF ( $O$ is Positive) AND ( $C$ is Neutral) & THEN NeighborDist is Far \\
\hline $\mathrm{R}_{9}$ & IF ( $O$ is Positive) AND ( $C$ is Positive) & THEN NeighborDist is Far \\
\hline$\ddot{R}_{44}$ & IF ( $E$ is Positive) AND ( $N$ is Neutral) & THEN PrefVelocity is Fast \\
\hline $\mathrm{R}_{45}$ & IF ( $E$ is Positive) AND ( $N$ is Positive) & THEN PrefVelocity is Fast \\
\hline
\end{tabular}

\subsubsection{Inference Method}

Three types of fuzzy inference methods are available: Mamdani, Larsen, and Takagi-Sugeno. In this paper, the Mamdani inference method [39], which includes aggregation, activation, and accumulation steps, is selected to calculate the fuzzy output of each decision parameter based on the Sup-Min composition.

The fuzzy rules proposed in Section 3.4.2 are a multidimensional multiple fuzzy reasoning model. The general format is as follows:

$$
\begin{array}{cccccc}
A_{11}, & A_{12}, & \cdots, & A_{1 n} & \rightarrow & B_{1} \\
A_{21}, & A_{22}, & \cdots, & A_{2 n} & \rightarrow & B_{2} \\
\vdots & \vdots & \cdots & \vdots & & \vdots \\
A_{m 1}, & A_{m 2}, & \cdots, & A_{m n} & \rightarrow & B_{m} \\
A_{1}^{*}, & A_{2}^{*}, & \cdots, & A_{n}^{*} \\
\hline \multicolumn{5}{c}{B^{*}}
\end{array}
$$

where $A_{i j}$ and $A_{j}^{*}$ are the fuzzy subsets of $U_{j} ; A_{i j}$ represents the $j$ th input of the $i$ th fuzzy rule in a fuzzy inference model; $A_{j}^{*}$ represents the $j$ th input of an actual antecedent; $B_{i}$ and $B^{*}$ are the fuzzy subsets of $V ; B_{i}$ represents the output of the $i$ th rule; $B^{*}$ represents the composite output of an actual antecedent $(i=1,2, \cdots, m ; j=1,2, \cdots, n) ; m$ is the number of fuzzy rules for a fuzzy inference model; and $n$ is the number of antecedent inputs of an 'IF-THEN' fuzzy rule. In this study, $V$ and $U_{j}$ are the universe of discourse of one decision preference and its corresponding personality factor, respectively.

The inference process is written as follows:

$$
\begin{gathered}
A_{1}(x)=\min \left\{A_{11}\left(x_{1}\right), A_{12}\left(x_{2}\right), \cdots, A_{1 n}\left(x_{n}\right)\right\} \\
A_{2}(x)=\min \left\{A_{21}\left(x_{1}\right), A_{22}\left(x_{2}\right), \cdots, A_{2 n}\left(x_{n}\right)\right\} \\
\vdots \\
A_{m}(x)=\min \left\{A_{m 1}\left(x_{1}\right), A_{m 2}\left(x_{2}\right), \cdots, A_{m n}\left(x_{n}\right)\right\} \\
A^{*}(x)=\min \left\{A_{1}^{*}\left(x_{1}\right), A_{2}^{*}\left(x_{2}\right), \cdots, A_{n}^{*}\left(x_{n}\right)\right\} \\
B_{1}^{*}(y)=\underset{x \in U}{\vee}\left[A^{*}(x) \wedge A_{1}(x) \wedge B_{1}(y)\right] \\
B_{2}^{*}(y)=\underset{x \in U}{\vee}\left[A^{*}(x) \wedge A_{2}(x) \wedge B_{2}(y)\right] \\
\vdots \\
B_{m}^{*}(y)=\underset{x \in U}{\vee}\left[A^{*}(x) \wedge A_{m}(x) \wedge B_{m}(y)\right] \\
B^{*}(y)=B_{1}^{*}(y) \vee B_{2}^{*}(y) \vee \cdots \vee B_{m}^{*}(y)
\end{gathered}
$$


where $x_{j}(j=1,2, \cdots, n)$ is the input value of the personality factor, and $B_{i}^{*}(y)(i=1,2, \cdots, m)$ is the intermediate result of each 'IF-THEN' rule, with $m=9$ and $n=2$. The operators $\wedge$ and $\vee$ take the minimum and maximum values of the membership functions, respectively; $B^{*}(y)$ represents a composite fuzzy set of output decision preferences.

\subsubsection{Defuzzification Method}

Because the consequences of the fuzzy rule set are also fuzzy subsets, these subsets must be transformed into crisp values through the defuzzification process. For defuzzification, several methods are available, including the mean of the maxima, average of the maxima, and centroid. Because of the accuracy of these methods, the most frequently used centroid method is adopted in this paper. The formula is given as follows:

$$
y_{\text {final }}=\frac{\int_{V} B^{*}(y) y d y}{\int_{V} B^{*}(y) d y}
$$

where $y_{\text {final }}$ is a final output of the fuzzy inference system, i.e., each one of the decision preferences.

In order to depict the relationship between inputs of personality factors toward the output of decision preferences, we show the surface plots of input and output of the fuzzy simulation in Figure 4 generated in Matlab.

a

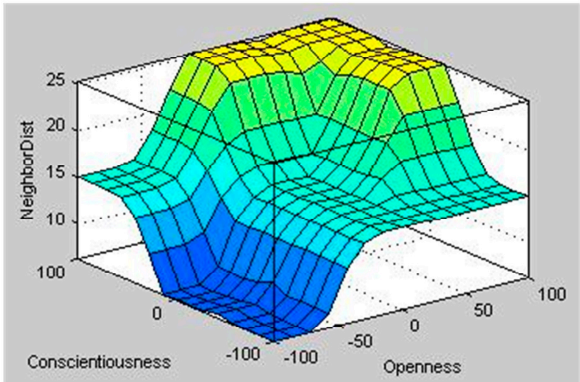

c

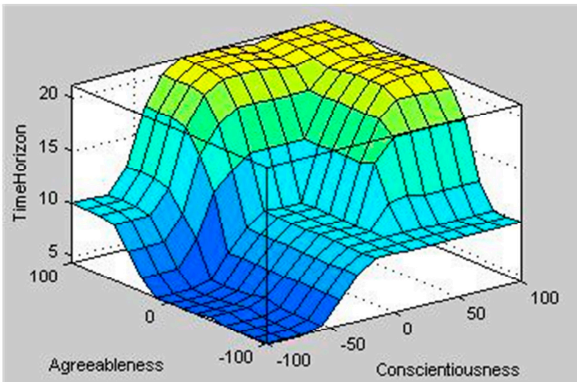

b



d

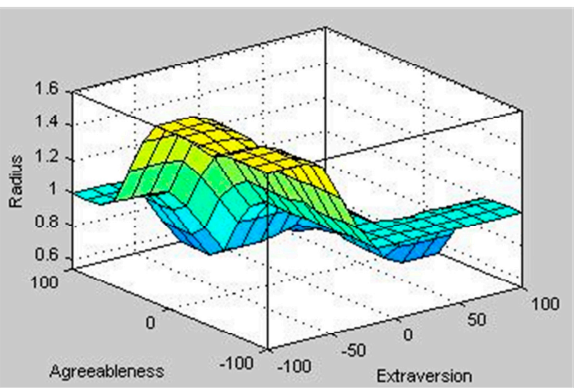

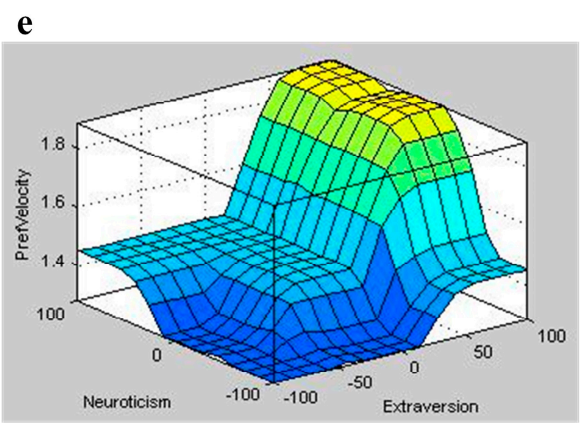

Figure 4. Surface plots of input and output of the fuzzy simulation (a) NeighborDist, (b) MaxNeighbors, (c) TimeHorizon, (d) Radius, and (e) PrefVelocity. 


\section{Simulation and Validation}

To test the proposed pedestrian model, we have developed a Visual C++ application based on publicly available Open GL and RVO2 [40] libraries to perform a variety of pedestrian simulation scenarios. All experiments are executed in real time by a PC (Dell OptiPlex 9020) with an Intel Core 3.6 GHz i7-4790 processor, an 8 GB memory and an AMD Radeon R5 240 graphics card with 1 GB memory. In this paper, we focus on the modelling and prediction of heterogeneous pedestrian dynamical behavior under normal or non-panic situations. Therefore, we simulated pedestrians with various personalities in several typical scenarios, including a single-exit room, a hallway with obstacles, and a narrowing passage. Furthermore, the effectiveness of our proposed model is validated by a user study. Although the distribution of personality traits in the population is closer to the normal distribution, we artificially set the distributions in our experiments, in order to obviously demonstrate different behaviors between heterogeneous pedestrians with various personalities.

\subsection{Simulation of Heterogeneous Pedestrians}

Figure 5 shows the motion trajectories taken by the highlighted agents in a single-exit room scenario. From left to right, the highlighted agents represent the pedestrians with positive (i.e., $V(i)=80$ ) Openness (blue), Conscientiousness (green), Extraversion (red), Agreeableness (orange), and Neuroticism (black) traits. The non-highlighted agents (grey) are given the default personality. The blue agent tends to choose more daring routes and takes a wavy trajectory. The green agent takes a fairly direct trajectory and moves in an orderly way behind the other agents. The red agent moves quickly and often tries to weave through others in the crowd. The orange agent can slightly adjust the direction of movement to avoid collision with the grey agents and thus takes a less direct trajectory. The black agent also moves quickly but takes a tortuous route when congestion occurs near the exit.

a



b

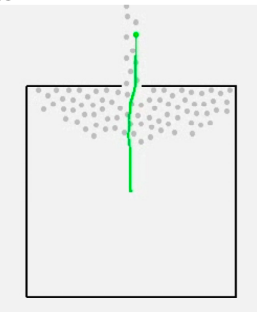

c

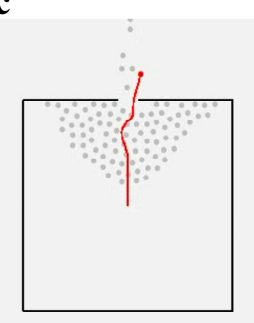

d

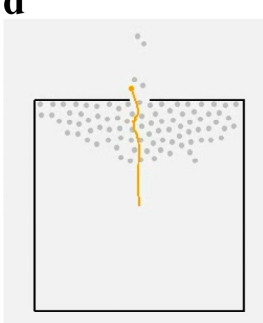

e

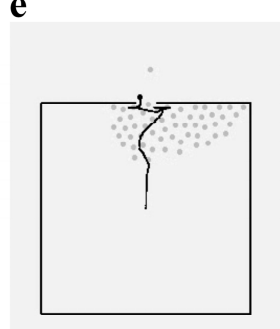

Figure 5. Motion trajectories of agents with default personality factors, although only one factor is positive. From left to right, the traits are (a) Openness, (b) Conscientiousness, (c) Extraversion, (d) Agreeableness, and (e) Neuroticism. All trajectories are displayed for an equal length of time.

With the same configuration above, Figure 6 demonstrates the different trajectories of five agents with distinct personality traits in the hallway with obstacles scenario. The agent with a positive Extraversion or Neuroticism trait can clearly be distinguished from the crowd. The agent with a positive Extraversion trait can pass through the crowd at a faster speed than others. The agent with a positive Neuroticism trait is easily deflected by the movement of the other agents. As for the remaining types, they take relatively straight trajectories and have no obvious differences under a non-congested situation. 


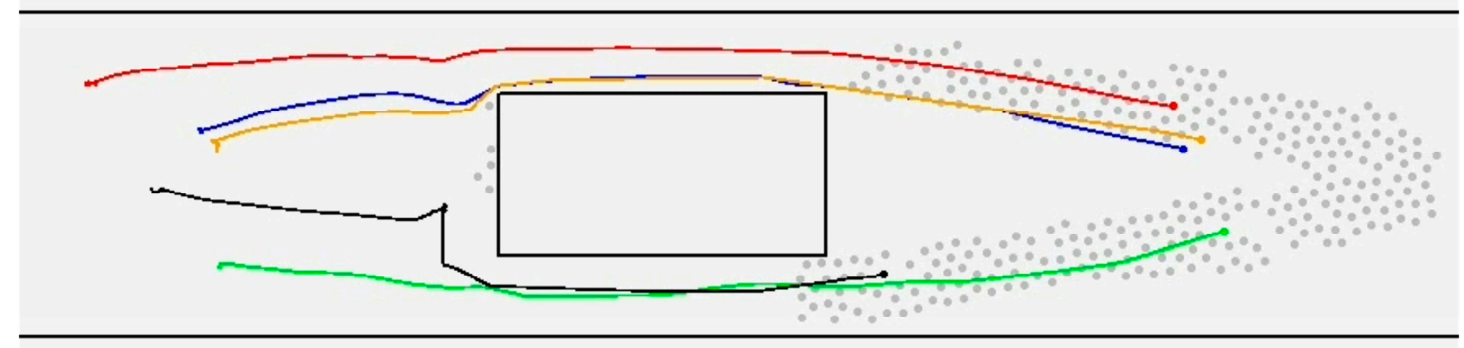

Figure 6. Comparison of motion trajectories among five agents with different personality types in the hallway with obstacles scenario. The agents with positive Openness (blue), Conscientiousness (green), Extraversion (red), Agreeableness (orange), and Neuroticism (black) traits are highlighted. The non-highlighted agents (grey) have the default personality traits.

In addition to comparing motion trajectories among agents with five personality traits, we have simulated the behavior of agents with a different level of each personality trait. Here, agents are simulated with a positive (i.e., $V(i)=80$ ) or negative (i.e., $V(i)=-80$ ) Extraversion trait in the narrowing passage scenario as an example. Figure 7 shows the results at the same time from two simulations in which the red agents are assigned a positive Extraversion trait on the left and a negative Extraversion trait on the right. Agents with a positive Extraversion trait tend to move quicker and closer to others than those with the negative trait. Furthermore, more agents simultaneously pass through the exit on the left. Agents with a positive Extraversion trait exit more efficiently in the narrowing passage than those with the negative trait.

a

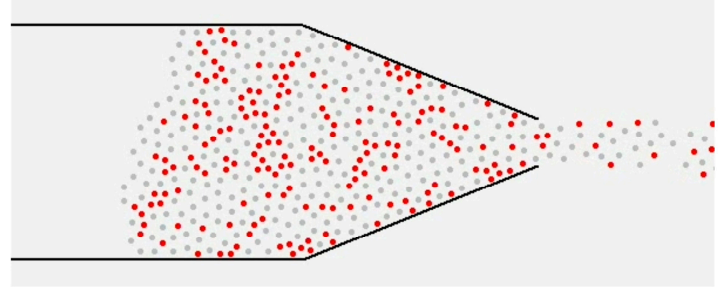

b



Figure 7. Comparison between (a) red agents with positive Extraversion and (b) red agents with negative Extraversion in the narrowing passage scenario for an equal length of time.

Figure 8 shows a comparison between homogeneous and heterogeneous pedestrian simulations in a single-exit room scenario. In the left simulation, all agents have the default personality. In the right simulation, the agents have a variety of personalities that are randomly generated. At the beginning of the two simulations, the agents are randomly distributed and have no significant differences as shown in Figure 8a. When the congestion phenomenon begins to occur (Figure 8b), the heterogeneous agents display various behaviors because of their different personality traits. For example, most of the agents with a positive Extraversion trait (red) walk at the front of the crowd and try to quickly exit by weaving through others. We can see from Figure $8 \mathrm{c}$ that the results of the homogeneous simulation are symmetrical and artificial when the congestion becomes serious. In contrast, the heterogeneous simulation is more reasonable and natural. In the final stage of the heterogeneous simulation as shown in Figure 8d, the agents with a positive Conscientiousness trait (green) are the majority of individuals who have not yet left the room. This result is consistent with the characteristics of self-disciplined pedestrians. 
a


c

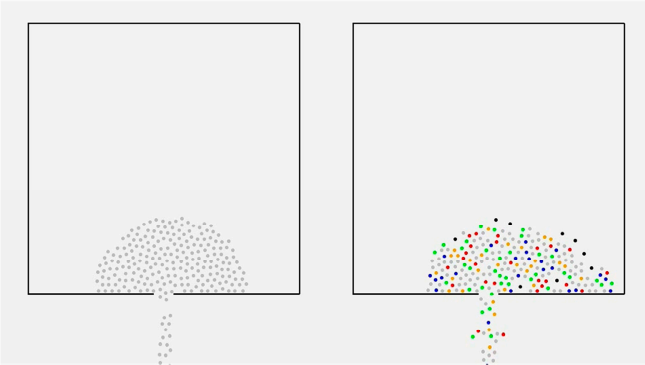

b

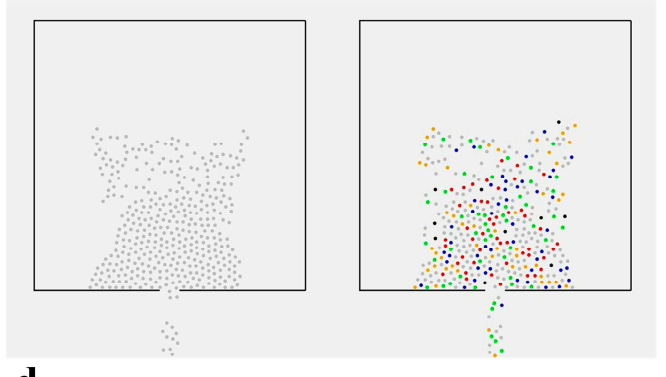

d

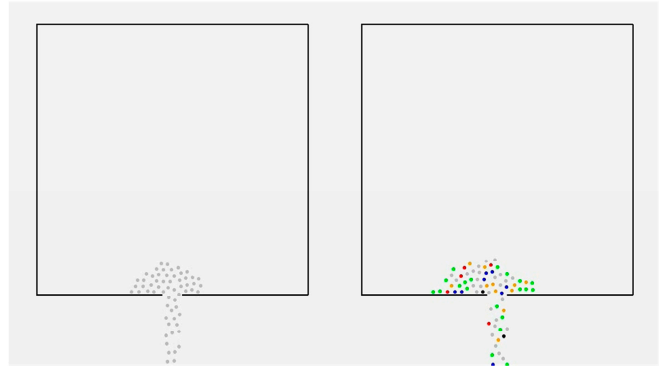

Figure 8. Comparison between homogeneous and heterogeneous pedestrians grouped by 200 individuals in a single-exit room scenario. The left panels show homogeneous pedestrians, and the right panels show heterogeneous pedestrians.

\subsection{Effect of Different Personalities During Evacuation}

To reveal how holistic crowd behavior is influenced by different personalities, we have simulated a pedestrian evacuation in a single-exit room by changing the percentage of pedestrians with a certain personality trait. Similar to the scenario described above, the scenario is a square room sized $20 \mathrm{~m} \times 20 \mathrm{~m}$ with a $1.5 \mathrm{~m}$ wide exit in the middle of the wall. The total number of agents is 200 , and their initial locations and directions are given at random in the room. Here, we choose the following five types as an illustrative example: including agents with positive Openness $(\mathrm{O}+)$, Conscientiousness $(\mathrm{C}+)$, Extraversion (E+), Agreeableness (A+), and Neuroticism (N+). Except for these agent types, the other agents all have the default personality.

In Figure 9, we plot the evacuation time versus the percentage of a certain type of agent in the crowd. The blue line indicates that the proportion of $\mathrm{O}+$ agents in the crowd has little effect on the total time of evacuation, and the green and orange lines show that with more $\mathrm{C}+$ or $\mathrm{A}+$ agents in the crowd, the evacuation can be more effective. The evacuation time decreases with an increasing proportion of $\mathrm{E}+$ agents in the crowd when the proportion is not very large. Nevertheless, the evacuation time increases if the proportion is larger than approximately $60 \%$. This result is related to the limitation of exit width and the occurrence of congestion, thus reflecting the well-known 'faster-is-slower' phenomenon. The black line shows that a greater proportion of $\mathrm{N}+$ agents leads to a significant increase in evacuation time. 


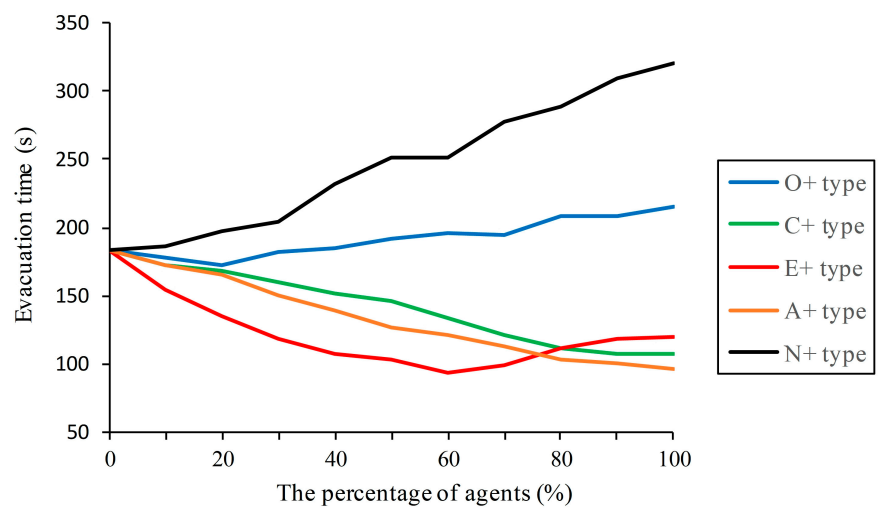

Figure 9. Evacuation time versus the percentage of certain types of agents in the crowd.

\subsection{Validation of the Proposed Model}

Evaluating the simulation results for pedestrian dynamical behavior influenced by human personality is a challenge. Here, the user study method is used to validate the proposed model because the real data are difficult to obtain and identify. This method has been proven feasible and effective by previous works $[5,10,11]$. A user study was completed by 72 participants ( 33 females and 39 males, ages 16 to 51) who had no previous knowledge of the experiment. Moreover, we repeated the same test three times to all participants, using new video examples for each time, to avoid testing them on best-case simulation scenarios only. Before the evaluation, participants were given a brief explanation of each factor of the OCEAN personality model. We designed a user study consisting of two sections (the first contains 5 questions and the second contains 10 questions).

The first section was designed to evaluate how well the different levels of each trait of the OCEAN model could be reproduced by our model. For each question, we created a pair of videos that simulate the movement of agents with a certain trait in the narrowing passage scenario. In each pair of videos, the simulations are generated by the highlighted agents with the positive and negative types of the trait. After watching the videos, the participants were asked to choose which simulation displayed the positive type trait in question. Figure 10 shows that the success rate for distinguishing between different levels of personality traits is high, especially for Neuroticism and Extraversion, which are 95.8\% and 94.4\% respectively, and the rates for Agreeableness, Conscientiousness and Openness are $81.9 \%, 79.2 \%$ and $73.6 \%$, respectively. To test the statistical significance of these results, we use a two-tailed binomial test to calculate the statistical $p$-values. The results indicate that the participants could correctly distinguish the positive and negative traits at a statistically significant rate $(p<0.05)$.

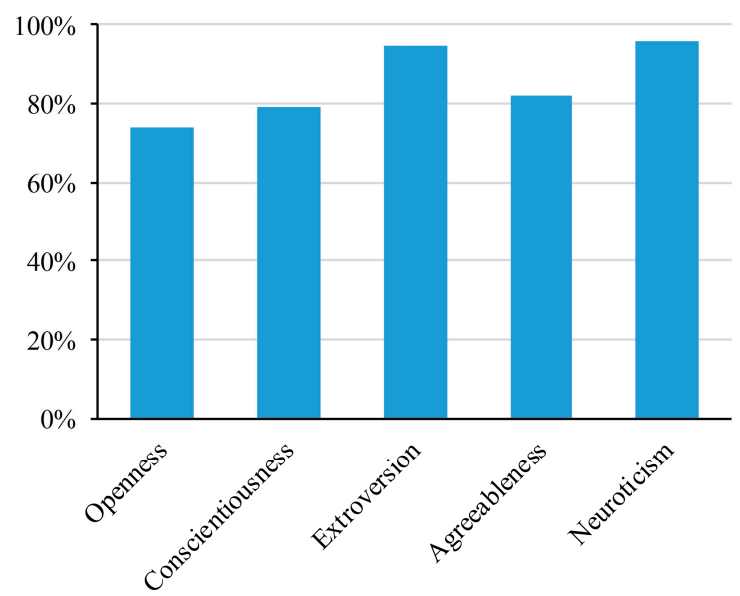

Figure 10. Success rate of distinguishing between the different levels of personality traits. 
The second section was intended to reflect the degree to which the five personality traits could be distinguished in the simulation by our model. All five positive traits have been compared with each other; thus, 10 videos presenting evacuation behavior in a single-exit room scenario were created. In each video, two highlighted groups of agents were assigned different personality traits (e.g., $\mathrm{O}+$ and $\mathrm{C}+$ ). In a corresponding question, the participants were asked to select which group was $\mathrm{O}+$ and which was $\mathrm{C}+$. The results are summarized in Table 5, which shows that most of the OCEAN personality traits could be correctly distinguished by the participants at a statistically significant rate $(p<0.05)$. Among these traits, the $\mathrm{E}+$ and $\mathrm{N}+$ traits were easily distinguished from the other traits. However, the participants had difficulty distinguishing between the $\mathrm{C}+$ and $\mathrm{A}+$ traits because of their similar characteristics in movement.

Table 5. Accuracy and $p$-values for distinguishing traits.

\begin{tabular}{ccc}
\hline Distinguishing Traits & Accuracy & $p$-Value \\
\hline O+ and C+ & $72.2 \%$ & $2 \times 10^{-4}$ \\
O+ and E+ & $78.8 \%$ & $2 \times 10^{-6}$ \\
O+ and A+ & $76.4 \%$ & $8 \times 10^{-6}$ \\
O+ and N+ & $87.5 \%$ & $4 \times 10^{-11}$ \\
C+ and E+ & $91.7 \%$ & $8 \times 10^{-14}$ \\
C+ and A+ & $66.7 \%$ & $6 \times 10^{-3}$ \\
C+ and N+ & $95.8 \%$ & $2 \times 10^{-15}$ \\
E+ and A+ & $79.2 \%$ & $7 \times 10^{-7}$ \\
E+ and N+ & $84.7 \%$ & $2 \times 10^{-9}$ \\
A+ and N+ & $95.8 \%$ & $2 \times 10^{-15}$ \\
\hline
\end{tabular}

\section{Conclusions}

In this paper, we propose a new pedestrian model that incorporates fuzzy logic theory into a multi-agent system to address cognitive behavior that introduces uncertainty and imprecision during decision-making. This model can describe the imprecise subjective decisions of a pedestrian while steering. Subjective decisions vary for pedestrians confronted with similar situations. To simulate heterogeneous pedestrians, the OCEAN personality model is introduced to model the psychological characteristics of a pedestrian, and it can generate the different decision preferences that represent the intrinsic control factors of decision-making. The fuzzy relationships between personality traits and decision preferences are determined by a fuzzy inference system. Finally, a variety of simulations and validation experiments are implemented in our developed application. The experimental results show that the proposed model can exhibit more reasonable and heterogeneous behavior in various scenarios and improve the credibility of the simulation; thus, it can be used to analyse how different personalities influence the crowd phenomena.

In the future, we intend to consider the aspect of physiological characteristics when simulating the heterogeneous pedestrian behaviors because pedestrian decision-making in movement is always subject to physiological conditions. Accordingly, the simulation effect of pedestrian dynamical behavior can be improved by combining psychological characteristics with physiological characteristics. Moreover, we intend to study pedestrian behavior in abnormal or unusual situations by adding an emotion model for the agents because emergent behavior can be profoundly influenced by instantaneous emotions.

Acknowledgments: This work was partly supported by the National Key Research and Development Program of China (Grant nos. 2016YFB0501502 and 2016YFB0501503), the Hainan Provincial Department of Science and Technology (Grant no. ZDKJ2016021), the Natural Science Foundation of Hainan (Grant no. 20154171) and the 135 Plan Project of Chinese Academy of Sciences (Grant no. Y6SG0200CX).

Author Contributions: Zhuxin Xue, Qing Dong, Xiangtao Fan, and Qingwen Jin conceived and worked together to achieve this work. Hongdeng Jian and Jian Liu performed the experiments. Zhuxin Xue wrote the paper.

Conflicts of Interest: The authors declare no conflict of interest. 


\section{References}

1. Treuille, A.; Cooper, S.; Popovic, Z. Continuum crowds. ACM Trans. Graph. 2006, 25, 1160-1168. [CrossRef]

2. Helbing, D.; Molnár, P. Social force model for pedestrian dynamics. Phys. Rev. E 1995, 51, $4282-4286$. [CrossRef]

3. Burstedde, C.; Klauck, K.; Schadschneider, A.; Zittartz, J. Simulation of pedestrian dynamics using a two-dimensional cellular automaton. Phys. A Stat. Mech. Appl. 2001, 295, 507-525. [CrossRef]

4. Helbing, D.; Farkas, I.; Vicsek, T. Simulating dynamical features of escape panic. Nature 2000, 407, 487-490. [CrossRef] [PubMed]

5. Cai, L.; Yang, Z.; Simon, X.; Qu, H. Modelling and simulating of risk behaviours in virtual environments based on multi-agent and fuzzy logic. Int. J. Adv. Robot. Syst. 2013, 10, 387. [CrossRef]

6. Brambilla, M.; Cattelani, L. Mobility analysis inside buildings using distrimobs simulator: A case study. Build. Environ. 2009, 44, 595-604. [CrossRef]

7. Berg, J.V.D.; Lin, M.; Manocha, D. Reciprocal velocity obstacles for real-time multi-agent navigation. In Proceedings of the IEEE International Conference on Robotics \& Automation, Pasadena, CA, USA, 19-23 May 2008; pp. 1928-1935.

8. Llorca, D.F.; Milanes, V.; Alonso, I.P.; Gavilan, M.; Daza, I.G.; Perez, J.; Sotelo, M.Á. Autonomous pedestrian collision avoidance using a fuzzy steering controller. IEEE Trans. Intell. Transp. Syst. 2011, 12, 390-401. [CrossRef]

9. Zheng, L.; Qin, D.; Cheng, Y.; Wang, L.; Li, L. Simulating heterogeneous crowds from a physiological perspective. Neurocomputing 2016, 172, 180-188. [CrossRef]

10. Durupinar, F.; Pelechano, N.; Allbeck, J.M.; Gudukbay, U.; Badler, N.I. How the ocean personality model affects the perception of crowds. IEEE Comput. Graph. Appl. 2011, 31, 22-31. [CrossRef] [PubMed]

11. Guy, S.J.; Kim, S.; Lin, M.C.; Manocha, D. Simulating heterogeneous crowd behaviors using personality trait theory. In Proceedings of the International Conference on Computer Graphics and Virtual Reality, Vancouver, BC, Canada, 5-7 August 2011.

12. Hughes, R.L. A continuum theory for the flow of pedestrians. Transp. Res. B Methodol. 2002, 36, 507-535. [CrossRef]

13. Reynolds, C.W. Flocks, herds and schools: A distributed behavioral model. ACM SIGGRAPH Comput. Graph. 1987, 21, 25-34. [CrossRef]

14. Pan, X.; Han, C.S.; Dauber, K.; Law, K.H. A multi-agent based framework for the simulation of human and social behaviors during emergency evacuations. AI Soc. 2007, 22, 113-132. [CrossRef]

15. Zadeh, L.A. Fuzzy sets. Inf. Control 1965, 8, 338-353. [CrossRef]

16. Lu, G.; Noyce, D.A. Pedestrian crosswalks at midblock locations: Fuzzy logic solution to existing signal operations. Transp. Res. Rec. 2009, 45, 63-78. [CrossRef]

17. Niittymaki, J.; Kikuchi, S. Application of fuzzy logic to the control of a pedestrian crossing signal. Transp. Res. Rec. 1998, 1651, 30-38. [CrossRef]

18. Nasir, M.; Nahavandi, S.; Creighton, D. Fuzzy simulation of pedestrian walking path considering local environmental stimuli. In Proceedings of the 2012 IEEE International Conference on Fuzzy Systems, Brisbane, Australia, 10-15 June 2012; pp. 1-6.

19. Zhu, B.; Liu, T.; Tang, Y. Research on pedestrian evacuation simulation based on fuzzy logic. In Proceedings of the 2008 9th International Conference on Computer-Aided Industrial Design and Conceptual Design, Kunming, China, 22-25 November 2008; pp. 1024-1029.

20. Li, M.; Li, S.; Liang, J. A fuzzy logic based approach for crowd simulation. In Advances in Electronic Commerce, Web Application and Communication, Volume 2 (Advances in Intelligent and Soft Computing); Jin, D., Lin, S., Eds.; Springer: Berlin/Heidelberg, Germany, 2012; pp. 29-35.

21. Dell'Orco, M.; Marinelli, M.; Ottomanelli, M. Simulation of crowd dynamics in panic situations using a fuzzy logic-based behavioural model. In Computer-Based Modelling and Optimization in Transportation; de Sousa, J.F., Rossi, R., Eds.; Springer: Cham, Switzerland, 2014; pp. 237-250.

22. Nasir, M.; Lim, C.P.; Nahavandi, S.; Creighton, D. A genetic fuzzy system to model pedestrian walking path in a built environment. Simul. Modell. Pract. Theor. 2014, 45, 18-34. [CrossRef]

23. Fu, L.; Song, W.; Lo, S. A fuzzy-theory-based behavioral model for studying pedestrian evacuation from a single-exit room. Phys. Lett. A 2016, 380, 2619-2627. [CrossRef] 
24. Zhou, M.; Dong, H.; Wang, F.Y.; Wang, Q.; Yang, X. Modeling and simulation of pedestrian dynamical behavior based on a fuzzy logic approach. Inf. Sci. 2016, 360, 112-130. [CrossRef]

25. McCrae, R.R.; Costa, P.T. Validation of the five-factor model of personality across instruments and observers. J. Personal. Soc. Psychol. 1987, 52, 81-90. [CrossRef]

26. Zheng, X.; Li, W.; Guan, C. Simulation of evacuation processes in a square with a partition wall using a cellular automaton model for pedestrian dynamics. Phys. A Stat. Mech. Appl. 2010, 389, 2177-2188. [CrossRef]

27. Blue, V.J.; Adler, J.L. Cellular automata microsimulation for modeling bi-directional pedestrian walkways. Transp. Res. B Methodol. 2001, 35, 293-312. [CrossRef]

28. Dai, J.; Li, X.; Liu, L. Simulation of pedestrian counter flow through bottlenecks by using an agent-based model. Phys. A Stat. Mech. Appl. 2013, 392, 2202-2211. [CrossRef]

29. Akasaka, Y.; Onisawa, T. Individualized pedestrian navigation using fuzzy measures and integrals. In Proceedings of the 2005 IEEE International Conference on Systems, Man and Cybernetics, Waikoloa, HI, USA, 12 October 2005; pp. 1461-1466.

30. Teknomo, K.; Gerilla, G.P. Fuzzy perceptional spacing for intelligent multi agent pedestrian simulation. In Proceedings of the International Symposium of Lowland Technology, Saga, Japan, 14-16 September 2006.

31. Pelechano, N.; Allbeck, J.M.; Badler, N.I. Controlling individual agents in high-density crowd simulation. In Proceedings of the 2007 ACM SIGGRAPH/Eurographics Symposium on Computer Animation, San Diego, CA, USA, 3-4 August 2007; pp. 99-108.

32. Balaji, P.G.; Srinivasan, D. An introduction to multi-agent systems. In Innovations in Multi-Agent Systems and Applications -1; Srinivasan, D., Jain, L.C., Eds.; Springer: Berlin/Heidelberg, Germany, 2010; pp. 1-27.

33. Ghasemaghaee, N.; Oren, T.I. In Towards fuzzy agents with dynamic personality for human behavior simulation. In Proceedings of the Summer Computer Simulation Conference, Montreal, PQ, Canada, 20-24 July 2003.

34. Phares, E.J. Introduction to Psychology, 3rd ed.; Harper Collins Publishers: New York, NY, USA, 1991.

35. Eysenck, H.J.; Eysenck, M.W. Personality and Individual Differences: A Natural Science Approach; Plenum Press: New York, NY, USA, 1985.

36. McCrae, R.R.; John, O.P. An introduction to the five-factor model and its applications. J. Personal. 1992, 60, 175-215. [CrossRef]

37. Goldberg, L.R. An alternative "description of personality": The big-five factor structure. J. Personal. Soc. Psychol. 1990, 59, 1216-1229. [CrossRef]

38. Berg, J.V.D.; Guy, S.J.; Lin, M.; Manocha, D. Reciprocal n-body collision avoidance. Springer Tracts Adv. Robot. 2011, 70, 3-19.

39. Mamdani, E.H. Application of fuzzy algorithms for control of simple dynamic plant. Proc. Inst. Electr. Eng. 1974, 121, 1585-1588. [CrossRef]

40. Berg, J.V.D.; Guy, S.J.; Snape, J.; Lin, M.; Manocha, D. RVO2 Library: Reciprocal Collision Avoidance for Real-Time Multi-Agent Simulation. Available online: http:/ /gamma.cs.unc.edu/RVO2 (accessed on 4 October 2017).

(c) 2017 by the authors. Licensee MDPI, Basel, Switzerland. This article is an open access article distributed under the terms and conditions of the Creative Commons Attribution (CC BY) license (http://creativecommons.org/licenses/by/4.0/). 